\title{
A Scheme to Identify Suspicious Activity on Video
}

\author{
Avuku Obulesh, Pogalla Rohini Reddy
}

\begin{abstract}
The structure is intended to help detect suspicious traffic authorities within the wide range of video information contained in the current inevitable observation video. reconnaissance inescapable this time. people talk about the thoughts and approaches to address near the hole of low-stage semantics between the motor and the complement is obvious from the video information and perception of human common. evaluation of the occasion depiction and techniques for utilising phrases which are recognised, and later on it is recommended that exam be carried out to enhance this shape. those examinations are applied as an knowledge to exchange statistics on the first-rate system you could rely upon whilst structuring a tale utilizing mechanical get collectively.
\end{abstract}

Keywords: video, viewer, mechanical editing.

\section{INTRODUCTION}

Large quantities of video surveillance data exist in today's world. Cameras are everywhere constantly recording daily occurrences from many angles. The main use for thisdataistoreviewtapeafteraknownevent

(e.g.abankrobbery)andgatherinformation of occasions (eg portrayal of the suspect) as a way to assist with wearing the culprits to fairness. anyways, if a video investigator who has no data on wherein and while, or irrespective of whether or not an occasion has struck make appropriate circulate turns out to be an increasing number of difficult. To find out an occasion suchasthis (ifitexists) ananalysthastosortthroughmanyhoursofvideotapeandmake an knowledgeable choice whether or not the events portrayed inside the video anticipate activity to be taken. protection protects (or different video examiner) can support a ton if there is no laptop framework can get acquainted with the events depending on the case of getting ready and use thisdefinitioninconjunctionwithsomeconfigurationparameter stodifferentiatebetween behavior "suspicious" and "regular". as an example, keep in mind the case of a protection protect checking video commentary corporation from the threshold fence. on the off danger that the gatekeepers suspect there may additionally have damaged the border fence sooner or later for the duration of the most latest forty eight hours, he have to bodily survey forty eight hours of recording for every digicam video recording perspectives wavering to discover infringement. As a median statement framework can also comprise handfuls or many digicam points, it immediately became out to be very dauntingtask.

Revised Version Manuscript Received on 30 May, 2019.

Avuku Obulesh, Anurag Group of Institutions , Hyderabad, Telangana, India. (Email : obulesh.a@gmail.com)

Pogalla Rohini Reddy, Anurag Group of Institutions , Hyderabad, Telangana, India.
An interface to the focus in the area of disconnected research might then be able to be created. on the hour of its specialists can survey whether move have to be made dependent on the video segment is featured. Inherently linked with this pastime is problemofbridgingthesemanticgapbetweenthelow-levelfeatu resamachineseeswhen avideoisinputintoit (e.g.shade, floor, form) andthehighlevelsemanticconcepts (or events) individuals see while seeing video cuts (eg, introductions, news discharges, boxingmatch).

Mechanical occasion of portrayal exploits the manner that a similar event stuck with the aid of an change digital camera preparations seem to be indistinguishable while predicted onto temporal dimension. Similar eventswhichdifferslightlyinscale, interpretation, evencolor rotationand (differentclothing) areclassifiedasthesameevent.Thischaracteristicallows ustoclusteravideointoevent-specificsegments, deviseaneventcomparisonmethod and symbolize "suspicious" movement for instance. The paper is to actualize the shape for the region association of suspicious events primarily based thistechnique

\subsection{The Data}

Each classifier will be fed a video sequence of length one to ten seconds featuring one of three events (walking, running, and waving). Approximately 20 video clips could be accrued and used to test every calculation. Video suggests every containing one Waving going for walks on foot jogging used to create occasion precis entertainer in opposition to the (generally) basis shifting to do one in every of 3 activities. one-of-a-kind entertainers inside the video display might be various sizes, sexual orientation and ethnicity and will put on an change outfit. Left to proper and proper to left instances of "directional" occasions, (for instance, on foot, going for walks) may be remembered for the video information. diverse foundations will likewise be utilized at some stage in the specific clasps. video information might be amassed using a Canon camcorder Z100

\section{2 methods}

Video records to be handled into four histogram portrayal utilising MatlabHistograms might be dwindled to the component vector vectorFeature of nineteen (out of 20) clasp will be applied for classifier educate (go away one pass approval) vector encompass the relaxation of the clasps can be utilized to test the name classifierOutput added by the 


\section{A Scheme to Identify Suspicious Activity on Video}

classifier will checked out towards the ground truth (manual marking) Repeat this progression for each video clipsCalculate classifier precision (right association/all models) Calculate the perplexity grid classifier (among activities)

\subsection{The Classifiers}

AI calculations to be assessed as a classifier on this evaluation are as in line with the following: Nearest Neighbor calculation (Histogram Distance feature) - this is a characterization calculation proposed by means of Zelnik-Manor et al. An underlying evaluation of its exhibition segment.Nearest itemized in beyond Neighbor calculation (Euclidean Distance) - This calculation ascertains the Euclidean separation among the whole thing of the thing vector and selecting Euclidean closest neighbor.

Nerves Networks-A perceptrons prepare containing 1024 statistics resources 1 yield and a few shrouded layer hubs. organized through lower back proliferation calculation.

the choice tree calculation is to construct a development of desire focuses dependent on highlights with negligible entropy

\subsection{Problem Definition}

Innately fix for this interest is an issue that includes an opening semantics a few of the functions of low-degree gadget to see whilst the video is including to it (eg, concealing, surface, form) and the concept of semantic nation atypical (or activities) an character appearance while looking portions (eg presentation, communicates, boxing organizes). There are procedures to control the attention of this problem need to recollect the low degree features will haul out, what chance need to nation and how unlabelled open doorways will begrouped. the main endeavor toward coping with this issue is to develop an photo delineating a warm event activities (the development of a progression of edges in the video) so that it can completely stand apart from numerous events.

\subsection{Motivation}

The utilization of the records is crucial for exam after the occasion recognized chronicle and accumulating information on that event so that it will help with conveying the blameworthy birthday celebration price. Regardless, if the specialist video that does not have facts on in which and whilst or whether or not threat had took place portrayed the progression from which turns as an increasing number of complex. As a ways as looking at suspects, there is probably a burst of the threshold of the fence sooner or later in the maximum latest forty eight hours, he would must be physically review the forty eight hours of movie for each camcorder seize the factors of view of the fence to find out the breaks. As a commonplace commentary system can join tens or multi-factor camera, it without delay transformed into a really extra exertion

\subsection{Objective}

The main aim towards solving the problem of overwhelming is to develop a robust event representation that characterizes the event (a series of frames in video sequence) to such an volume that it tends to be definitively contrasted and distinctive activities.
The objective is to attend to the difficulty by way of coming across like-which low-level highlights may be extricated, how the activities need to be spoken to and the way the activities might be ordered unlabelled case

\section{LITERATURE REVIEW}

Erich Schikuta et al. [1] constructed up "A Framework Execution Grid-Clustering technique" for group examination. The method has confirmed precious for exploratory records exam and moreover fundamental to the strategies for records mining. The goal of bunch examination is to discover a courting of room and to isolate the estimation of the records esteems into a whole lot of regarded from the sooner of subgroups depending on comparison measurements. due to excessive-dimensional data,

ie records with an full-size variety of trends depict, grouping can end result into a totally tedious task, which frequently restrict the quantity of perceptions to be bunched practically speaking. To determine this difficulty, Grid amassing technique has been created, which perform comparability esteems do now not figure the estimation of their information, but set the advantage of encompassing area records esteems, as an instance by the document of a particular information shape.

on this paper, the creators gift a system that makes it manageable to assess the diverse records systems for the age of multi-dimensional machine structure collecting the statistics esteems into the rectangular. those features are then accrued by means of neighbor search calculation based totally on a rectangular structure topology. because the major statistics shape record structure BANG assessed gift and display its attainability as a bunching list. Propelled gadget is meant to accept as a bundle to the product Weka.

in this paper, a structure for lattice based bunching calculation is delivered. This framework takes into consideration the usage and investigate multi-dimensional list structure various as the reason for the grouping calculation, which delivered about the parcel of the space an incentive to the structure in their files. within the last form the consumer can have the likelihood to reflect onconsideration on and pick between distinct systems of the record. As of currently BANG report shape is executed, as brought in this paper. a few systems are extra multi-dimensional within the focal factor of studies later on, because the R-tree, kd-timber, and so on. The framework has been finished can be reachable in two paperwork: an impartial rendition with distinct UI and data manipulate and module bundle, that's coordinated into the Weka circumstance.

event grouping and discovery strategies Lavee Gal et al. [3] made an investigation of "A Framework for evaluation Video device for Detection of Suspicious events" which proposes a device for investigators within the video assist detectingsuspicious movement of a variety of video records that exist in this point in time of video observation throughout. The thoughts and tactics to close the semantic hollow between low-stage system meaningful highlights of the video information and great stage occasion visible via

Published By:

Blue Eyes Intelligence Engineering 
human spectators are examined. assessment of the occasion and phraseology order techniques delivered and future endeavors to improve this system is proposed. those trials applied as a result in a conversation at the most perfect AI calculations to get acquainted with the schematic portrayal suggests that proposed in this paper. The proposed framework took another video grouping named and marked a few video successions communicate to diverse kinds of activities manageable. It creates the belief of the substance in the video arrangement marked as a yield to the purchaser. clients can modify this illustration as per their dispositions (ie what type of activities they view as suspicious) using an interface Video evaluation device.

The occasion version portrayal to correctly quantify the sum and form of modifications that show up in a scene beneath the suspicion that a similar occasion, (for example, walking, taking walks, waving) could have a similar conveyance adjustments for every one of the three measurements considered. A video occasion is spoken to as a lot of $\mathrm{x}, \mathrm{y}$ and $\mathrm{t}$ slope strength histogram for a few transient scales. scale fleeting granularity used to display the motion of an event (eg in high-granularity timescale community movement as arm swing when taking walks could be captured at the same time as the low-scale worldly granularity will seize the whole challenge within the area movement). for example, take four distinct worldly scales each with a massive portion of the quantity of edges as the only going before it. After standardization of the histogram territory last step is smoothed esteems. this is done to restrict the affects of neighborhood crisscrosses towards the correlation work. that is completed by using strolling down the histogram and setting each really worth canister with verage estimation of muddle in his neighborhood (generally 5 receptacles on the 2 aspects). while this strategy promises some relics, as an instance, slopes to the excellent angles, has been confirmed a hit in increasing the size of the large territories inside the histogram correlation

\subsection{Data Mining Techniques}

Berkhin Pavel et al. [4] "have a look at of Clustering facts Mining strategies" directed an exam that gathering is a department of statistics into gatherings of a comparable object. speakme to the information with less organization could lose certain exceptional subtleties, however accomplishing disentanglement. This statistics version with its bunch. information demonstrating to put a meeting in an genuine factor of view is established in arithmetic, measurements, and numerical examination. From the factor of view of AI institution as in line with the concealed examples, discovering bunches is unaided learning, and the following framework is the idea of facts. From the viewpoint of all the way down to earth collecting assumed a noteworthy process in records mining programs, as an instance, logical investigation of facts, information recuperation and content material mining, spatial database programs, web examination, CRM, promoting, restorative diagnostics, computational technological know-how, and severa others.

Grouping is a subject of dynamic research in a few fields, as an instance, insights, design acknowledgment, and AI. The overview focused on grouping in data mining. information mining consist of inconveniences gathering full-size datasets with amazingly manyattributes of different sorts. This forces a one in every of a type computational necessities on the full-size bunching calculation. specific calculations have evolved as of late that meets those stipulations and efficaciously implemented to authentic problems of statistics mining. they're the difficulty of opinions.

\section{PROPOSED METHODOLOGY}

The Fig.2 gives the description of the proposed methodology.

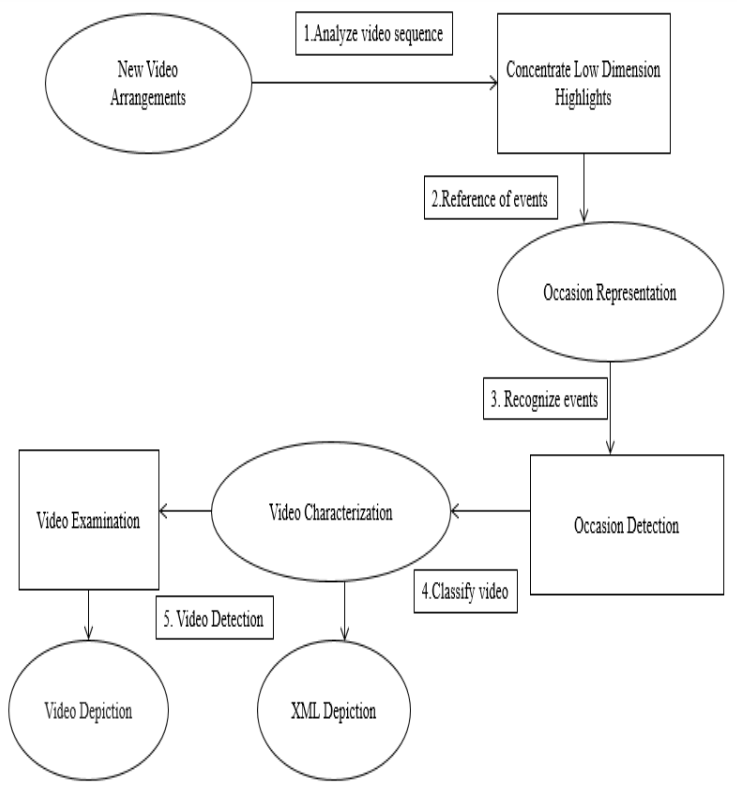

Fig.2: Framework of the proposed methodology.

The idea of co-clustering of data points and attributes is old and is known under the names simultaneous clustering, bi-dimensional clustering, block clustering, conjugate bunching, grouping move, and strategies for statistics bottleneck. The utilization of duality for the examination of hetero out data (double or multidimensional scaling) moreover has a long records in insights. A comparative approach is to collect a gathering of factors introduced within the Co-prevalence of categorical records. inside the utility it'd reflect the force of the reaction characteristics in tissue exams, recurrence of go to motion of a web page, or various in-keep deals in keeping with category of merchandise

\subsection{Weighted Feature Selection}

A have a look at led by way of Lei Wang et al. [5] "Programmed photo Annotation and Retrieval using weighted function selection" portrays that, mechanical enhancements added approximately an widespread quantity of non-literary statistics, as an example, photos. photo explanation talented and recuperation framework is fantastically fascinating. grouping calculation makes it manageable to talk to the visible highlights of photos with boundless photographs. In view of this, a super deal of real models, which ruin down the correspondence between the visible highlights and the words and find the shrouded semantics, were disbursed. This model builds the 


\section{A Scheme to Identify Suspicious Activity on Video}

clarification and taking a large photograph database. Be that as it may, the photograph statistics regularly has infinite measurements. traditional grouping calculations dole out a similar load to these measurements, and turn out to be befuddled throughout the time spent coping with those measurements. in this paper, we endorse a weighted detail choice calculation as a solution for this issue. For a selected bunch, determine the pertinent highlights depending on histogram exam and dole out extra noteworthy load to the crucial highlights contrasted with less relevant highlights. they have completed a wide variety of models to interface visible token with watchwords depending on the aftereffects of k-implies bunching calculation with a weighted detail choice and without spotlight desire, and assessed execution using exactness, evaluation and precision of correspondence using benchmark datasets

\subsection{Clustering Algorithms}

Rui Xu et al. [7] "have a look at of Clustering set of rules" represents that records investigation assumes a critical job to recognize an collection of marvels. bunch research, investigation crude with next to zero earlier information, comprising of studies created in unique networks. assorted variety, from one standpoint, outfits us with severa instruments. then again, reasons disarray picked calling. amassing calculation to the informational series up in measurements, software program engineering and AI, and delineates their software in a few benchmark informational collections, voyaging income rep trouble, and bioinformatics, the energizing new field of concentrated endeavors. some themes are firmly associated, the region measure, and approve the organization, are likewise mentioned. As a widespread apparatus for facts investigation, marked bunch breaks down analyzed the statistics, both by way of constructing a diverse leveled shape, or installation a number of gatherings as indicated with the aid of the quantity of preespecified. The process consists of a progression of steps, beginning from the pre-getting ready and calculation development, and evaluation of the legitimacy of the association. every firmly diagnosed with one another, and it offers an incredible check to train. right here, putting the eye on bunching calculations and checking on distinctive procedures confirmed up inside the writing. those calculations evolved from various studies networks, looking ahead to to take care of various problems, and have their very personal upsides and drawbacks. Albeit as of now found severa instances of effective use of institution examination, still many open troubles due tothe nearness of numerous questionable factors intrinsic. these issues have pulled in and could preserve on drawing within the focused endeavors of the expansive orders. they are condensed and finished up the evaluation with a rundown of some key problems and patterns inquire about for organization calculation

\subsection{Subspace Clustering Algorithm}

Lei Wang et al. [8] conducted a research on "Automatic Image Annotation and Retrieval Using Subspace Clustering Algorithm". The development of technology delivered limitless non-literary information, as an example, pictures. picture explanation gifted and recovery framework is profoundly captivating. bunching calculation makes it manageable to talk to the visible highlights of photographs with boundless photographs. In mild of this, a outstanding deal of actual fashions, which spoil down the correspondence among the visible highlights and the phrases and locate the concealed semantics, were disbursed. This version builds the explanation and taking a big photograph database. Be that as it can, the photo information typically has limitless measurements. commonplace bunching calculations allocate a similar load to these measurements, and emerge as confounded during the time spent coping with these measurements. in this paperauthors advocate top-down, subspace bunching calculation as an answer for this trouble. For a specific organization, determine the critical highlights depending on histogram research and dole out greater noteworthy load to the applicable highlights contrasted with much less enormous highlights. they have actualized four particular fashions for connecting visible token with watchwords dependent on the outcomes of grouping calculation and ok-implies bunching calculation, and assessed execution making use of exactness, overview and precision of correspondence utilizing benchmark datasets. The outcomes indicated that the proposed calculation is superior to standard ones for programmed image depiction and restoration

\subsection{Corr-LDA}

A pay attention through David M. Blei et al. [14] "Commented on Modeling statistics" is considered as a demonstrating issue data with severa sorts in which instances of 1 type, (as an example, subtitles) fills in as a portrayal of different kinds, (for example, pictures). They painting 3 numerous leveled probabilistic blend version that means to depict the information, completing in correspondence idle Dirichlet allotment, dormant variable fashions of powerful showing joint dissemination of the 2 sorts and conveyance of the clarifications given restrictive primary kinds. They led investigates Corel photograph database and data, survey the presentation concerning

of held-out ability effects, programmed clarifications, and content based totally photograph retrievalThe creators have created Corr-LDA, an terrific model to the information depicted that joins the upsides of probabilistic grouping for measurement decrease with an specific model of wherein the restrictive appropriation of statistics - coming about documentation. in the setting of the image statistics/subtitle, has indicated that this model can accomplish an competitive and possibly with unequalled contingent conveyance given word photograph

\subsection{Statistical Distance Measure}

Zelnik-Manor L. et al. [17] directed studies on "investigation of event-primarily based Video" suggests that dynamic activities can be considered as a protracted haul common items, portrayed by spatio-transient highlights at extraordinary worldly scales. In mild of this, they dependent a honest actual proportion of the separation between the video groupings (possibly of numerous period) in light of the substance of their conduct. This progression is a 
non-parametric and in this manner can cope with an assortment of dynamic events. They make use of this degree to restrict and represent events in a continual video grouping lengthy. This turned into managed without a in advance records at the type of event, their model, or to the extent their worldly. The aftereffects of the order manner is a fleeting department of video successions to reveal constant lengthy sub-arrangements, and their amassing into a dependable bunch occasion.

activities recognized with the portrayal and degree the separation can likewise be applied for event primarily based ordering to long video preparations, in any occasion, while most effective one quick example cut gave. anyhow, whilst multiple instances of events that equivalent clasp gave (both because of the grouping manner, or appointed bodily), it has a tendency to be applied to improve the portrayal of the occasion, the dimensions of the separation in question, and as per the nature of reputation and bunching procedure. Having occasion based totally separation degree among the preparations, may be utilized to disconnect and symbolize occasions in a continual video grouping long. This was controlled and not using a earlier facts on the type of event, their version, or to the volume their herbal discern

\subsection{Texture Images and Statistical Learning}

A concentrate by means of Ziv Bar-Joseph et al. [18] "mixing Texture and Texture Synthesis utilising Statistical gaining knowledge of movie" prize dependent on genuine getting to know calculations to comprise static and time-fluctuating floor that matches the statistics floor look. The proposed calculation is a fashionable and programmed, and features admirably on an assortment of surfaces including 1D floor of sound, image floor 2nd and three-D films. A comparable approach is likewise used to create $2 \mathrm{~d}$ surface blended on the same time capture the presence of various different enter floor. in this methodology, the floor of the data is dealt with as a examined sign produced by using a stochastic system. First form a multi-scale revolutionary treerepresenting of sign utilizing wavelet exchange. From this tree, the tree every other abnormal produced by way of gaining knowledge of and trying out the restrictive likelihood of music in specific tree

\subsection{Spatio-Temporal Motion}

Yong Rui and P. Anandan [19] proposed "interest Segmentation primarily based visual Spatio-Temporal styles motion". examination of human activities caught within the video succession has come to be a topic of intrigue is important in pc imaginative and prescient. A substantial part of the past paintings has concentrated on the difficulty of acknowledgment of acts or physical games, yet dismissing the problem of recognizing the points of confinement of the pastime in a video succession that carries visible act ordinary and self-assertive. This paper presentations a way to address this trouble by using spotting discontinuities temporary spatial examples of movies that seize the pastime. They talk to an part to define regarding optical-flow coefficient to a brilliant extent large head component is decided from the entirety of the movement field inside the given video grouping. At that factor pick out worldly discontinuities in the route of this coefficient depends on three unique sizes. At long final, they contrasted with their fragment limit recognizable with the aid of human eyewitnesses in a comparable request in the research of mind research late autonomous human view of visual occasions. The effects indicated the trial brings approximately the two groupings applied in this examination. The exploratory results are promising both visual evaluation and contrasted with the results of intellectual studies.

\section{RESULTS AND DISCUSSION}

Temporal scale 1 is usually composed of all frames in the video sequence. For example, if Video Sequence V1 is 120 frames long then the intensity value matrix of V1

- at temporal Scale 1 includes frames $1,2,, \ldots .120$ (120 frames)

- at temporal Scale 2 includes frames $1,3,5, \ldots .120(60$ frames)

- at temporal Scale 3 includes frames $1,5,9, \ldots .120$ (30 frames)

- at temporal Scale 4 includes frames $1,9,17, \ldots 120(15$ frames)

Once these 3D intensity value matrices are obtained for each temporal scale it can be able to calculate the intensity gradient for each of the three directions. The force inclination esteem quantifies the adjustment in power in each one of the three headings $(x, y, t)$. Histogram of these characteristics constitute the dissemination of the complete scene modifications. a total portrayal of an occasion is twelve 256 histogram canister (one for every $(\mathrm{x}, \mathrm{y}, \mathrm{t})$ the measurements in extra of four scale fleeting). The inclination for each size is determined by the everyday contrast between the estimation of the present and next/past incentive to the precise dimension. determine 2 suggests this manner for 2-dimensional grid.

\subsection{Extracting the Event Representation}

Video portions that communicate to the occasion and casting off $3 \mathrm{D}$ grid computing the pressure slope of those traits within the $\mathrm{x}, \mathrm{y}$ and heading for each one of the four worldly scales. At lengthy closing, this variation those traits by way of taking their very best traits and standardized inclination vector of length 1 .

interim mixed waste covers the complete run force perspective esteem. This histogram, on this manner, could be very primary for the plot, imagine and examine. Histogram is determined by using taking the scope of 12 community is normalized inclination ( $\mathrm{x}, \mathrm{y}, \mathrm{t} * 4$ scale worldly) and separate it into 256 equivalent receptacles. A check of the attitude esteems that fall in each this sort of containers is an incentive inside the histogram canister listing. y-hub compares to the quantity of pixels whose angle esteem falls into a particular canister and the $\mathrm{x}$-hub relates to the field list. After standardization of the histogram location remaining step is smoothed esteems. this is completed to limit the impacts of close by confounds towards the correlation paintings. it's miles with the aid of taking walks down the histogram and placing each field esteem by means of the ordinary estimation

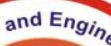

? IJTRE 


\section{A Scheme to Identify Suspicious Activity on Video}

of muddle in his community (usually 5 canisters on the two facets).

\subsection{Event Comparison}

12 histogram portrayal of the video fragments served evaluation them with portrayals of others to decide if the 2 talk to a kind of multiplied degree semantic ideas (occasions). because of this a honest degree the separation squared circumstance has been dependent, in which $\mathrm{L}$ is the amount of fleeting scale (4 in this test) and h (I) is the histogram field esteem at document $\mathrm{I}$. $\mathrm{k}$ is various measurements $(\mathrm{x}, \mathrm{y}, \mathrm{t})$ and 1 talking to four diverse brief scales.

$$
D^{2}=\frac{1}{3 L} \sum_{k, l, i} \frac{\left[h_{1 k}^{l}(i)-h_{2 k}^{l}(i)\right]^{2}}{h_{1 k}^{l}(i)+h_{2 k}^{l}(i)}
$$

This measure averages the difference between each bin in the histogram of each dimension of each temporal scale over the total number of histograms. This produces a number that indicates how close the two compared events are to one another.

\subsection{Event Detection}

A good event representation and event comparison function allows us to begin to detect and classify events in a new video sequence. However, the process determining which video frames constitute an event and how to separate adjacent events inside the video grouping nevertheless requires definition. occasion vicinity problem, thus, is characterized in awesome publications on this paper. the first is a rearranged problem. in this trouble has given video arrangement containing a solitary occasion. To characterize these activities utilizing this portrayal plot and the correlation work. inside the $2 \mathrm{nd}$, step by step mind boggling, translation of the difficulty become given a video arrangement containing an difficult to understand degree of activities and can decide the activities and in which (what's the threshold range) within the video they show up.

The underlying advance on this technique for the second one shape of this trouble is a lot of video quantities (and their histogram portrayal) and bodily arranging this. at least any such "Classifiers", as named them, all there is possibles occasion within the scene. to enhance the precision of positive classifiers speak to a comparable sort of event must be of various lengths, take place in numerous geographic zones of the scene and mirror the heading of each improvement. in the "straightforward", one event for every video element, issue every other event is grouped using a classifier with the call of closeness nearest to him (the littlest separation esteem).

\subsection{XML Video annotation}

in the wake of encountering video grouping location activities portrayed in the beyond phase of activities contained in it are placed away in XML position. because this arrangement is whatever but difficult to gadget meaningful examiners are presently prepared to address the video information is notably greater effective (making use of the Video evaluation tools). XML likewise saw discernible and consequently the human customer content material synopsis of occasions can likewise create a respectable portrayal of a fraction of the video being noted. The XML report consists of a reference to the video records facts, video qualities express fragments, (for example, video period and document arrangements), and information on any occasion that happens within the video component. activities Video displayed as puzzling additives and sub-components included, for instance, occasion type, event start point and length of the occasion. XML document is a explanation of the electricity at a few destiny date be supplanted with an all the extra dominant computer group affordable (eg, video VEML occasion that cosmology language). this will permit a greater profound degree of portrayal in case of video sections by using the use of the connection cardinality, which means of setting and distinctive highlights accessible in the metaphysics language.

\subsection{A Video Analysis Tool}

Accessibility of information device intelligible clarification, if this is in XML or particular video content philosophy language layout, a tremendous increase toward connecting the semantic hollow. A video exam gadgets that accept this sort of clarification as facts and set the appropriate video portions touching on direction viable. Such an utility should fill in as a guide to manipulate examiners search for "suspicious" activities in the video facts movement. The occasion pulled in may be characterized progressively by using investigators for going for walks the software. The definition will at that factor be contrasted and the video factors and a comparable side can be set apart for in addition investigation. however, the expert can parent out what is viewed as everyday behavior and every event depicted stray from this widespread may be stamped.

Investigators will at that factor don't forget every one of the fields set apart to decide if interest is required. A shading covered scroll bar (with an intriguing video sections is shown by means of an exchange shading) may be utilized as a graphical interface for this errand. professionals could then be capable of moreover refine the inquiry parameter with bogus and proper tremendous imprint.

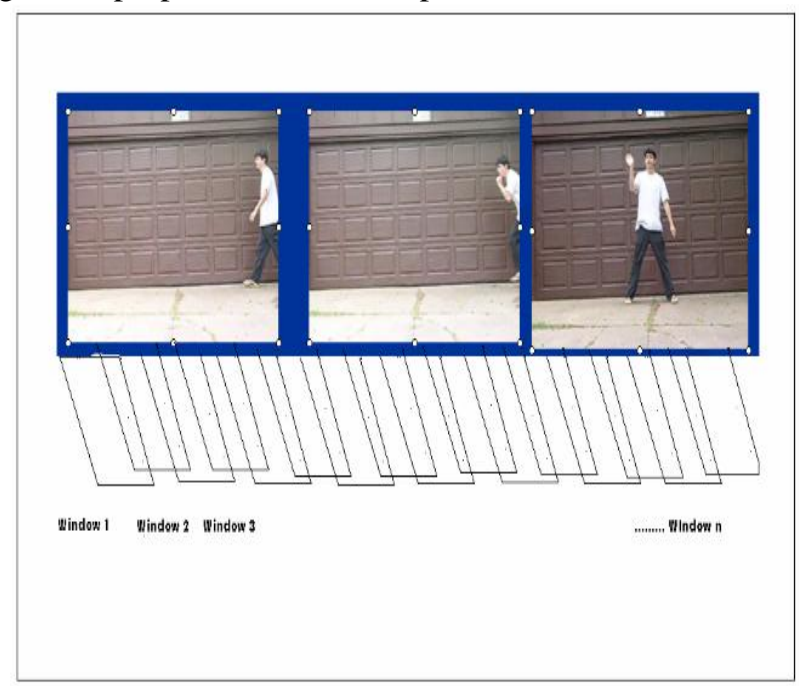

Fig.3: Detection of Events in a Video Sequence. 


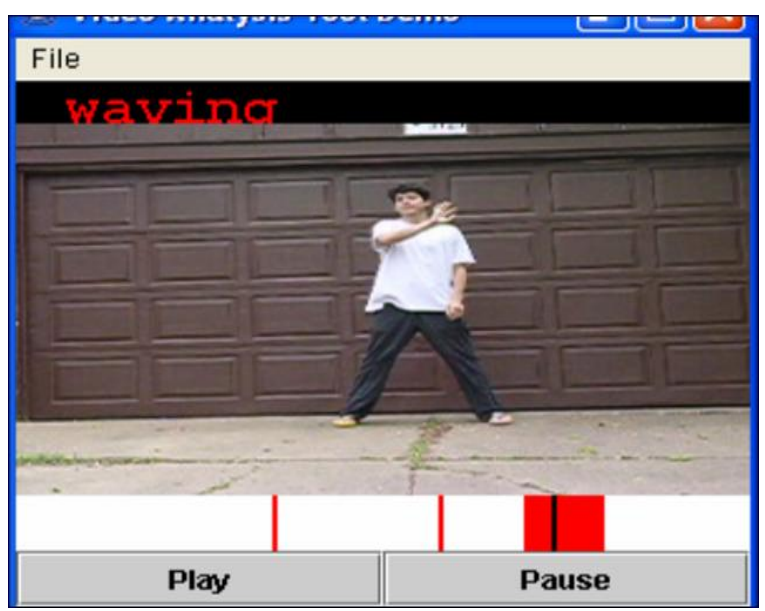

Fig. 4: screen shot from a prototype.

\section{CONCLUSIONS}

The paper presented the results of some preliminary experiments were conducted to gauge the potential of this schema. The The aftereffects of this preliminary have been promising but more interior and out investigation should be achieved earlier than giving judgment on the portrayal, gaining knowledge of and grouping plans which might be talked about in this paper. consequently, the investigation of the proposed trials will differentiate the presentation of diverse gaining knowledge of calculations and determine if it could enhance modern systems. This exam likewise gave a few speculative results of such an evaluation and differentiation the features and shortcomings of different calculations.

\section{REFERENCES}

1. Erich Schikuta and Florian Fritz, "An Execution Framework for Grid-Clustering method", international convention on Computational science, quantity eighty, 2016.

2. Benjamin S Duran and Patrick L Odell. "group analysis: review", quantity 100. Springer technology and enterprise Media, 2013.

3. Gal Lavee, Latifur Khan, Bhavani Thuraisingham, "A structure for video exam apparatuses for the area of suspicious activities" Multimedia tools and programs October 2007, volume 35, issue 1, pp 109-123.

4. Pavel Berkhin, "an outline arranges statistics mining methods", in the multidimensional data bunching, Springer, 2006.

5. L. Wang and L. Khan, "Programmed image Annotation and Retrieval utilising function choice weighted Multimedia tools and programs", Kluwer Publishers, January seventeenth, 2005.

6. Ian H Witten and Eibe Frank, "statistics Mining: sensible AI devices and methods", Morgan Kaufmann, 2005.

7. Rui $\mathrm{Xu}$, Donald Wunsch, "evaluation accumulating calculation", Neural Networks, IEEE Transactions on, sixteen (three): 645-678, 2005.

8. L. Wang, L. Liu and L. Khan, "Programmed picture Annotation and Retrieval the usage of Subspace Clustering set of rules," in Proc. ACM MMDB, Arlington, Virginia, November 2004.

9. Jia-Yu Pan, Hyung-Jeong Yang, Pinar Duygulu, and Christos Faloutsos, "Programmed photo text", In proceedings of the 2004 IEEE international convention on Multimedia and Expo (ICME 2004) 2004.

10. C. Ordonez and E. Omiecinski, "An powerful plate based k-implies for social databases," IEEE Trans. Knowl. statistics Eng., Vol. sixteen, no. eight, pp. 909-921, August 2004 